\title{
Estrategia didáctica para enseñar a dirigir el proceso de enseñanza-aprendizaje de la resolución de problemas matemáticos
}

Pérez González, Andel; Quero Méndez, Ortelio Nilo; Bravo Viera, Jorge Luis

Estrategia didáctica para enseñar a dirigir el proceso de enseñanza-aprendizaje de la resolución de problemas matemáticos

Revista Educación, vol. 45, núm. 1, 2021

Universidad de Costa Rica, Costa Rica

Disponible en: http://www.redalyc.org/articulo.oa? $\mathrm{id}=44064134007$

DOI: https://doi.org/10.15517/revedu.v45i1.42112

Esta obra está bajo una Licencia Creative Commons Atribución-NoComercial-SinDerivar 3.0 Internacional. 


\title{
Estrategia didáctica para enseñar a dirigir el proceso de enseñanza-aprendizaje de la resolución de problemas matemáticos
}

\author{
Andel Pérez González \\ Universidad de Sancti Spiritus "José Marti Pérez", Cuba \\ apgonzalez@uniss.edu.cu \\ (iD http://orcid.org/0000-0003-4435-4030 \\ Ortelio Nilo Quero Méndez \\ Universidad de Sancti Spiritus "José Martí Pérez", Cuba \\ oquero@uniss.edu.cu \\ (iD) http://orcid.org/0000-0002-7872-2957 \\ Jorge Luis Bravo Viera \\ niversidad de Sancti Spiritus "José Marti Pérez", Cuba \\ jlbravo@uniss.edu.cu \\ (iD) http://orcid.org/0000-0001-9861-7743
}

A Didactic Strategy for Teacher Training Leadership for Math Problem Resolution

DOI: https://doi.org/10.15517/revedu.v45i1.42112

Redalyc: http://www.redalyc.org/articulo.oa?id=44064134007

Recepción: 08 Junio 2020

Aprobación: 05 Agosto 2020

\section{Resumen:}

El presente escrito, resultado del proyecto de investigación en que participan sus autores, describe la estrategia didáctica elaborada con el fin de enseñar a dirigir el proceso de enseñanza y aprendizaje de la resolución de problemas a estudiantes de las carreras Licenciatura en Educación, Matemática-Física y Licenciatura en Educación Matemática. Su diseño, guiado por la utilización de una metodología cuantitativa, consideró la fundamentación teórica asumida en relación con la formación didáctica y los resultados del diagnóstico aplicado al estudiantado de las mencionadas carreras. En la estrategia, se explicitan de manera integrada las acciones que deben efectuarse desde las diferentes disciplinas y, también, desde los componentes académico, laboral e investigativo en función de la formación didáctica para la resolución de problemas.

Palabras Clave: Estrategia didáctica, Formación didáctica, Proceso de enseñanza y aprendizaje de la Matemática, Resolución de problemas, Enseñanza-Aprendizaje.

\section{Abstract:}

This article shares the results of a study by the authors describing didactic strategies developed to train students majoring in Education, Physics-Math or Math Education. The study design is based on a quantitative methodology which considers the theoretical foundation of didactic training. The results of the diagnosis were analyzed in the context of the students in the aforementioned majors. Strategies include actions to be executed from various disciplines as well as academic, labor and research components according to the type of didactic training used for problem resolution.

KEYWORDs: Didactic Strategy, Didactic Training, Mathematics Teaching and Learning Process, Problem Resolution, TeachingLearning.

\section{INTRODUCCIÓN}

La sociedad contemporánea exige procesos formativos que respondan a las realidades del contexto y a las transformaciones que caracterizan las relaciones políticas y sociales. Al respecto, organizaciones científicas reconocen el rol de la educación en la preparación de las actuales y futuras generaciones para la resolución de los problemas que deberán enfrentar en su vida diaria. 
Ante esta realidad, se hace necesario que las universidades presten especial atención a la formación del profesional de la educación. De ahí que, en este artículo, se priorice la formación didáctica del estudiantado de las carreras pedagógicas; por ser la dimensión que distingue su modo de actuación.

En tal sentido, se identifica que una de las tareas más complejas del proceso de formación de los profesionales de la educación es, sin lugar a duda, preparar al estudiantado para dirigir el proceso de enseñanza y aprendizaje. Paradójicamente, es esta una aspiración ineludible para los centros formadores; de ahí, que los investigadores en didáctica de la Matemática reflexionen sistemáticamente en torno a la interrogante: ¿Cómo lograr la formación didáctica del futuro profesor de Matemática?

En particular, se centra la atención en la preparación de los futuros profesores de Matemática, para la dirección del proceso de enseñanza y aprendizaje de la resolución de problemas. Es por ello que el objetivo del artículo es proponer una estrategia didáctica que contribuya al perfeccionamiento del proceso de formación didáctica de estudiantes, futuros profesores de Matemática, para la resolución de problemas.

$\mathrm{Su}$ desarrollo estuvo guiado por una metodología predominantemente cuantitativa que exigió la utilización de métodos teóricos como el histórico-lógico, el analítico-sintético y el inductivo-deductivo; los cuales permitieron analizar y construir las posiciones teóricas que fundamentan el diseño de la estrategia didáctica. Igualmente, se utilizaron métodos empíricos como el análisis del producto de la actividad y la observación; estos facilitaron la recogida y el análisis de los datos que ilustraron el estado actual de la formación didáctica del alumnado de las carreras con que se trabaja. Durante el proceso de la investigación, sus actores principales fueron estudiantes y docentes del colectivo pedagógico de las carreras antes mencionadas.

El artículo se organiza en cuatro apartados: el primero, caracteriza el estado actual de la formación didáctica para la resolución de problemas; el segundo, presenta los fundamentos teóricos relacionados con la formación didáctica del profesorado de Matemática; el tercero, relaciona los fundamentos de la estrategia didáctica como resultado científico y, en el cuarto, se describe la estrategia didáctica como resultado del estudio que posibilita la solución de la problemática que origina la investigación.

\section{Estado de la formación didáctica para la resolución de problemas, en estudiantes futuros docentes de Matemática}

Una vez precisado el objetivo del artículo y al considerar la importancia de la formación didáctica para el profesional de la educación, se procedió a desarrollar un diagnóstico del nivel de formación didáctica para la resolución de problemas, en estudiantes de las carreras Licenciatura en Educación Matemática-Física (en fase de culminación) y Licenciatura en Educación Matemática. En tal sentido, se tuvieron en cuenta las dimensiones e indicadores propuestos por Pérez et al. (2018).

Para obtener información relevante sobre la problemática de investigación, se aplicaron métodos cuantitativos y cualitativos; entre ellos: la encuesta, la observación, la entrevista en profundidad, la revisión de documentos y la prueba pedagógica.

La población estuvo integrada por la matrícula total de ambas carreras y la muestra se conformó por once estudiantes; de ellos nueve (tres de $4^{\circ}$ año y cuatro de $5^{\circ}$ año del curso diurno y dos del $4^{\circ}$ año curso encuentro) de la primera carrera y dos de la segunda (de $2^{\circ}$ año, modalidad curso encuentro).

La medición del constructo resultó de la evaluación directa de las dimensiones, y estas a su vez de la obtenida al evaluar cada indicador. En todos los casos se utilizó una escala de cinco valores: muy adecuado, bastante adecuado, adecuado, poco adecuado e inadecuado. El valor final de cada indicador, dimensiones y del constructo se obtuvo mediante el cálculo del promedio (p) al que posteriormente se le asignó un valor de la escala antes mencionada. Es decir, inadecuado para $\mathrm{p}<1,5$; poco adecuado para $\mathrm{p}<2,5$; adecuado para $\mathrm{p}<3,5$; bastante adecuado para $\mathrm{p}<4,5$ y muy adecuado si $4,5<\mathrm{p} \leq 5$. La triangulación de la información de 
los indicadores permitió evaluar las dimensiones y el constructo que se estudiaba; sus resultados se ilustran en la Tabla 1.

TABLA 1

Categoría final de los indicadores, las dimensiones y el constructo evaluado

\begin{tabular}{|c|c|c|c|c|c|c|c|}
\hline Dimensión & Indicadores & Promedio & Categoría & Promedio & Categoría & Promedio & Categoría \\
\hline & 1 & 3.23 & A & & & & \\
\hline & 2 & 2.94 & A & 3.02 & A & & \\
\hline \multirow[t]{7}{*}{1} & 3 & 2.90 & $\mathrm{~A}$ & & & & \\
\hline & 1 & 3.18 & A & & & & \\
\hline & 2 & 3.82 & $\mathrm{BA}$ & & & & \\
\hline & 3 & 2.48 & PA & & & & \\
\hline & 4 & 2.20 & PA & 2.78 & A & & \\
\hline & 5 & 2.51 & $\mathrm{~A}$ & & & & \\
\hline & 6 & 2.48 & PA & & & 2.85 & A \\
\hline \multirow[t]{4}{*}{2} & 7 & 2.76 & A & & & & \\
\hline & 1 & 2.78 & A & & & & \\
\hline & 2 & 2.64 & A & & & & \\
\hline & 3 & 2.84 & $\mathrm{~A}$ & 2.72 & A & & \\
\hline \multirow[t]{3}{*}{3} & 4 & 2.65 & A & & & & \\
\hline & 1 & 3.06 & A & & & & \\
\hline & 2 & 3.06 & $\mathrm{~A}$ & 2.96 & A & & \\
\hline 4 & 3 & 2.76 & A & & & & \\
\hline
\end{tabular}

Fuente: Elaboración propia

El análisis cuantitativo de los resultados permitió plantear como principales hallazgos las fortalezas y debilidades que caracterizaban la formación didáctica para la resolución de problemas en estudiantes de ambas carreras.

Entre las fortalezas se identifica que generalmente el alumnado:

- Domina las acciones del procedimiento para resolver problemas matemáticos.

- Logra un desarrollo adecuado de la habilidad resolver problemas matemáticos (siempre que los contenidos se corresponden con los programas escolares).

- Identifica los procedimientos heurísticos y las etapas del programa heurístico general como herramientas para la resolución de problemas.

- Muestra interés por aprender a resolver problemas y por apropiarse de las herramientas matemáticas y didácticas necesarias para enseñar a resolverlos.

Igualmente, constituyen debilidades del estudiantado las siguientes:

- En ocasiones, presenta insuficiencias en el dominio y la comprensión del significado de algunos de los conocimientos matemáticos escolares.

- Evidencia un limitado desarrollo de habilidades matemática básicas que resultan de obligatoria aplicación al resolver problemas matemáticos.

- Con frecuencia, desconoce las estrategias de aprendizaje a utilizar para enseñar a resolver problemas.

- Frecuentemente, olvida tener en cuenta las exigencias metodológicas de la línea directriz relacionada con la resolución de problemas al trabajar con estos.

- Generalmente, muestra limitaciones para integrar los contenidos matemáticos y didácticos al desarrollar el tratamiento metodológico de los problemas.

Estos resultados evidencian una contradicción entre estado real y el estado deseado del nivel de formación didáctica para la resolución de problemas, en las personas participantes del estudio. De ahí, que sea necesario 
profundizar en los referentes teóricos a considerar durante el diseño de la estrategia didáctica integradora que se propone como vía de solución a la problemática objeto de análisis en la investigación.

\section{Marco teórico para la formación didáctica del profesorado de Matemática}

Los investigadores en didáctica de la Matemática consideran como elementos relevantes aquellos que hacen que la formación didáctica sea distintiva y acorde a las particularidades de los contenidos matemáticos. Es por ello, que a continuación se reconocen las posiciones más influyentes que caracterizan su evolución y se precisan aquellas que los autores del artículo asumen.

La formación didáctica del profesorado de Matemática ha sido considerablemente desarrollada desde significativos espacios de debate científico. En el contexto internacional, el estudio centra la atención en las ideas debatidas en los Congresos Internacionales de Matemática; en el primero de ellos, Otte (1979) reconoció la necesidad de vincular la teoría y la práctica para el logro de la formación didáctica de los futuros docentes de Matemática.

En posteriores ediciones, Cooney (1986) exigió la integración de los componentes del proceso de formación en función de la formación didáctica. En estos espacios, se resalta la caracterización del conocimiento profesional del profesorado que se deriva de los trabajos de Shulman (1986). Este autor, alega que el cuerpo docente tiene un conocimiento del contenido, un conocimiento curricular y un conocimiento didáctico del contenido.

Dado el interés del estudio, se profundiza en el conocimiento didáctico del contenido; el cual se identifica como

las formas más corrientes de representar un contenido, las analogías más poderosas, ilustraciones, ejemplos, explicaciones y demostraciones -en una palabra, las formas de representar y formular el contenido para hacerlo más comprensible a otros(...). La comprensión de lo que hace que determinado tópico sea fácil o difícil; las concepciones y preconcepciones que más frecuentemente tienen los estudiantes, y los marcos en que se aprenden (Shulman, 1986, p. 13).

Estas posiciones se comparten por los autores del artículo. Las más significativas defienden la formación didáctica como los procederes o modos de actuación que con mayor seguridad permiten enseñar los contenidos matemáticos.

Sobre la formación didáctica, Rico (1992) destacó la relación entre la didáctica de la Matemática y el dominio del contenido matemático; para este autor, solo el dominio integrado de ambos contenidos asegura el ejercicio eficiente de la docencia. Asimismo, Oliveras (1997) sugirió que como parte de la formación didáctica había que enseñar a formular interrogantes, a generar aprendizajes matemáticamente ricos y a diseñar modelos de aprendizaje según las condiciones. Ambas posiciones, resultan de interés para el presente estudio.

Según los criterios anteriores, Gómez (2000) explica, la importancia de la didáctica de la Matemática para la temática, y González (2000), afirma que un desempeño de calidad requiere de cambios en su proceso de enseñanza y aprendizaje.

Ambos insisten en que la formación didáctica del profesorado de Matemática implica saber hacer comprensible sus contenidos a partir de analogías, ilustraciones, ejemplos y contraejemplos, explicaciones, demostraciones y formas de representación de los diferentes temas. Se comparten sus criterios, aunque se enriquecen según las tendencias actuales que caracterizan la enseñanza y el aprendizaje de la asignatura.

En este mismo sentido, Gómez (2000), al indagar sobre la formación del profesorado de Matemática, subraya que en España se han conjugado dos ideologías con incidencia directa en la formación didáctica: una, que supone que para enseñar es suficiente con el dominio de la disciplina y otra, que distingue la didáctica como un arte y como tal, el profesorado se forma dentro de su propia práctica, o guiado por los prácticos. 
Desde el primer punto de vista, se señala que lo importante es la formación científica y, por tanto, se reniega de lo didáctico por ser vista como una ciencia que va en detrimento del conocimiento disciplinar. Desde el segundo punto de vista, se entiende que la Didáctica debe centrarse en la instrucción y en la práctica; es decir, en los problemas que enfrenta el conjunto docente al dirigir el proceso de enseñanza y aprendizaje; posición esta que se comparte.

De acuerdo con Godino (2002), el estudio de esta temática exige de un conocimiento suficiente del contenido disciplinar al que se refieren dichos conocimientos didácticos. Resultan interesantes sus planteamientos referidos a la didáctica de la Matemática, en particular, cuando alega que esta debe propiciar sentido al lenguaje, los conceptos y métodos matemáticos por parte del alumnado, mediante su referencia a las situaciones y problemas matemáticos presentes en la vida.

También, este autor destacó tres alternativas para la formación de docentes de Matemática, la primera centra la atención en la formación matemática, la segunda jerarquiza la formación didáctica ya que presupone que el estudiantado conoce suficientes matemáticas y la tercera trata de manera integrada ambas formaciones; posición esta última más defendida en la actualidad en países de América y Europa.

A partir de esta reflexión, Godino y Batanero (2003) valoran la importancia de la integración de los conocimientos de la didáctica de la Matemática en función del logro de una adecuada formación didáctica de los futuros profesores de esta asignatura.

De forma similar, para Flores y Peñas (2003) el profesorado debe propiciar que el estudiantado logre una representación de su futura tarea profesional, que la vea como un educador que emplea las matemáticas para ayudar al crecimiento de los sujetos. Al respecto, plantea que, para ello se necesita de un bagaje de conocimiento didáctico, basado en análisis teóricos y contrastados con experiencias empíricas. Desde esta posición, se logra que el conocimiento didáctico sea significativo para el estudiantado.

Sobre la misma temática, Llinares y Krainer (2006) precisan la pertinencia de lograr, desde la enseñanza y el aprendizaje de la didáctica de la Matemática, una práctica reflexiva que conduzca a aprender sobre la enseñanza y a evaluar sus resultados como sinónimo de una elevada formación didáctica. Por otra parte, un hito importante en la evolución del tema lo constituyó el modelo desarrollado por Ball, Thames y Phelps (2008) quienes proponen que el conocimiento matemático que utiliza el profesorado en el aula para producir instrucción y crecimiento en el alumnado es analizado como el conocimiento matemático para la enseñanza.

Godino y Batanero (2008), al profundizar en sus criterios explicitan que para la formación didáctica del profesorado de Matemática es necesario centrar la atención en el conocimiento matemático y el didáctico. En otros estudios, Godino (2009) analiza los tipos de conocimientos que debe dominar un/a profesor/a de Matemática; al respecto, destaca los didácticos-matemáticos dentro del cual se incluye el didáctico, el matemático y las competencias del profesorado para saberlo trasmitir y enseñar a sus estudiantes a partir del conocimiento de estos.

Posteriormente, el propio Godino (2011) expresa que la didáctica de la Matemática describe y explica el proceso de enseñanza y de aprendizaje y orienta su acción efectiva hacia la práctica a la vez que promueve su mejora a partir de enfocarse hacia el logro de una formación didáctica de los futuros profesores de Matemática.

En igual medida Sierra, Bosch y Gascón (2012) destacan que la formación didáctica exige integrar las cuestiones relativas a la enseñanza de la Matemática. Desde su perspectiva, consideran que se trata de promover un aprendizaje que sea el resultado de una vivencia o situación práctica, de que el estudiantado viva un acontecimiento similar a los muchos que vivenciará en el día a día de su profesión. Para ello, es necesario establecer los vínculos entre el desarrollo de la ciencia didáctica, el desarrollo del sistema de enseñanza y la formación de sus agentes. De ahí, la necesidad de lograr una formación didáctica que integre ambos saberes.

En los trabajos de Pino-Fan y Godino (2015) se reconoce que el conocimiento matemático para la enseñanza está conformado por dos grandes categorías, cada una de las cuales, a su vez, está conformada por otras subcategorías del conocimiento: 
- Conocimiento del contenido, que incluye conocimiento común del contenido, conocimiento especializado del contenido y conocimiento en el horizonte matemático.

- Conocimiento pedagógico del contenido, conformado por el conocimiento del contenido y el estudiantado, conocimiento del contenido y de la enseñanza y conocimiento del currículo.

Los autores anteriores, citando a Schoenfeld y Kilpatrick (2008) proponen distinguir los siguientes contenidos de la formación didáctica: conocer las matemáticas escolares con profundidad y amplitud; conocer a las y los estudiantes como personas que piensan; conocer a las y los estudiantes como personas que aprenden; diseñar y gestionar entornos de aprendizaje; desarrollar las normas de la clase y apoyar el discurso de la clase como parte de la enseñanza para la comprensión; construir relaciones que apoyen el aprendizaje y reflexionar sobre la propia práctica. En estas posiciones, a juicio de los autores del artículo, se explicita la necesaria integración de los contenidos matemáticos y didácticos que necesita un/a profesor/a de esta asignatura.

Del estudio elaborado se precisa la importancia de relacionar los contenidos a la práctica, de hacer comprensible los programas escolares y de promover la reflexión de las acciones de planificación y ejecución. También destaca como tendencia más defendida la que insiste en formar un/a docente que domine los contenidos matemáticos y didácticos y los integre al dirigir el proceso de enseñanza y aprendizaje.

Seguidamente, se analiza el tema desde el contexto cubano. Al respecto, se afirma que son insuficientes los trabajos que se refieren específicamente a la formación didáctica. A pesar de ello, se consideran aspectos que detallan su importancia y actualizan sus contenidos al ser vistos desde la didáctica de la Matemática.

Sobre este particular, Ballester et al. (1992) plantean que el estudiantado debe adquirir los conocimientos sobre los objetivos, la estructuración del contenido, el trabajo con los programas, así como desarrollar habilidades para la dirección del proceso de enseñanza y aprendizaje y, entre ellas, destacan la planificación.

En tal sentido, Fernández (1999) apunta que el no declarar las habilidades profesionales a lograr constituye una limitación con incidencia directa en la formación didáctica del profesorado de Matemática; aspecto, que en cierta medida aún prevalece, pues las declaradas tienen un elevado nivel de generalidad. En esta temática, profundiza Pérez (2015) al estudiar la formación y el desarrollo de la habilidad profesional planificar el proceso de enseñanza y aprendizaje de la Matemática.

Por otra parte, Gort (1999) insiste en la pertinencia de lograr el vínculo con la práctica para comprobar las teorías pedagógicas y didácticas estudiadas, y destaca la necesidad de establecer relaciones interdisciplinarias, particularmente con las dedicadas al estudio de los contenidos matemáticos y didácticos.

Almeida (2000) destaca la necesidad de enseñar a desarrollar al futuro profesorado de Matemática las tareas correspondientes a la función docente-metodológica. En su propuesta realza el papel de la disciplina Didáctica de la Matemática y su contribución al cumplimiento de los objetivos del año, al integrar los componentes, al promover la participación y el carácter de problema de las tareas docentes dirigidas a la planificación; aspectos que influyen en la concepción actual del proceso de formación didáctica del cuerpo docente de Matemática.

Según Feria (2003), una adecuada formación didáctica requiere asumir el aprendizaje como un proceso de construcción de significados donde cobran vida los problemas profesionales, los objetivos, los contenidos y los métodos de la ciencia a enseñar, idea que permite comprender el papel de la didáctica en la formación inicial.

A tono con esta posición, Garcés (2003) propone una concepción didáctica para la estructuración del proceso de enseñanza y aprendizaje de la Matemática; esta propuesta concibe la unidad de la formación matemática y la formación didáctica en función del modo de actuación profesional y centra la atención en el trabajo con el sistema de tareas, elemento de trascendental importancia para la formación didáctica.

Se coincide con este investigador cuando afirma que la pedagogización de la Matemática se presenta como un fenómeno exclusivo de la disciplina Didáctica de la Matemática, pues no se enfatiza en el papel que les corresponde a todas las disciplinas y a todos los componentes de este proceso en función de la formación didáctica. 
Al respecto, Beltrán (2012) destaca la pertinencia de atender con prioridad la planificación del proceso de enseñanza y aprendizaje, y la identifica como una de las habilidades profesionales de interés. Argumenta que, si una persona docente aspira, por lo menos, a un mínimo de calidad en su gestión docente-metodológica, debe planificar cada actividad docente por efectuar. Sin embargo, no profundiza en el cómo enseñar a planificar el proceso de enseñanza y aprendizaje de la Matemática.

En tal sentido, como ya se precisó antes, Pérez (2015) desarrolla una caracterización de la habilidad profesional planificar el proceso de enseñanza y aprendizaje de la Matemática, contentiva de una conceptualización de tres habilidades de menor nivel de generalidad: analizar metodológicamente unidades, diseñar sistemas de clases y planificar clases, así como la precisión de las acciones y operaciones que deberá aprender a ejecutar el futuro profesorado de esta asignatura para cada una de ellas.

Finalmente, se reconoce que la Didáctica de la Matemática aporta los elementos teóricos y prácticos necesarios para aprender a dirigir el proceso de enseñanza y aprendizaje. Sin embargo, es importante comprender que para lograr una adecuada formación didáctica del personal docente de Matemática es imperativo enfatizar en la integración de las disciplinas del plan de estudio y en el aprovechamiento de las potencialidades de los componentes y escenarios en que se desenvuelve la formación profesional.

Al tener en consideración los criterios estudiados, los autores plantean las posiciones teóricas que fundamentan la formación didáctica para la resolución de problemas. Por lo significativo que resulta para el diseño de la estrategia didáctica que se describe, a continuación, se resumen los más importantes.

Primeramente, se asumen los criterios de Pérez, Rodríguez y Quero (2017b) al considerar la formación didáctica como

la preparación que deben adquirir los estudiantes para dirigir el proceso de enseñanza y aprendizaje; la cual se expresa en la apropiación y aplicación integrada de los conocimientos, habilidades y actitudes profesionales propios de la ciencia a enseñar y de la didáctica general y particular; así como en el sistema de experiencias profesionales relacionadas con la planificación, ejecución y el control del proceso de enseñanza y aprendizaje (p. 4).

Segundo, serán atendidas las exigencias que plantean Pérez et al. (2017a) para lograr una acertada formación didáctica del estudiantado de la Licenciatura en Educación Matemática, en ellas se reitera la necesidad de:

- Lograr, desde las tareas docentes, la integración necesaria de los componentes laboral, académico e investigativo en función del cumplimiento de los objetivos.

- Desarrollar las habilidades comunicativas a través de la argumentación de ideas y propuestas de tratamiento metodológico a contenidos matemáticos.

- Desarrollar la independencia cognoscitiva a partir de promover la búsqueda y el procesamiento de información y la utilización de diferentes fuentes.

- Promover la elaboración y solución de ejercicios y problemas para luego explicar cómo utilizarlos en una clase o sistema de clases.

- Sugerir la confección de instrumentos para el diagnóstico de los conocimientos y las habilidades de una unidad, o instrumentos de evaluación sistemática y parcial.

- Orientar la preparación del análisis metodológico de unidades, de sistemas de clases y de clases de contenidos seleccionados.

- Argumentar el enfoque metodológico de la asignatura a partir de los contenidos de las diferentes áreas de la Matemática (p. 3).

De igual manera, se consideran orientaciones metodológicas que fundamentan el proceso de formación didáctica algunas de las ideas expresadas en los documentos normativos de la carrera Licenciatura en Educación Matemática; seguidamente se relacionan los más importantes para el diseño de la estrategia que se propone. En el modelo del profesional (Ministerio de Educación Superior de Cuba, 2016) se plantea que 
los problemas profesionales pedagógicos constituyen una guía para la formación de pregrado de los educadores. Así se denominan a aquellas situaciones inherentes al objeto de la profesión que deberán resolver los educadores durante el desempeño de su labor educativa profesional (p. 6).

La idea anterior confirma la posición de los autores de insistir en la necesidad de que el proceso de formación didáctica se caracterice por un enfoque problémico y profesional; ya que una de las situaciones profesionales a las que con frecuencia se deberá enfrentar el alumnado es precisamente la resolución de problemas.

Por otra parte, en el propio documento (MES, 2016) se precisan los problemas profesionales generales y específicos para los cuales se debe preparar el estudiantado y a los que se contribuye con cada actividad del proceso de formación profesional. Las siguientes ideas se relacionan con los que más aportan a la formación didáctica para la resolución de problemas:

- La dirección grupal del proceso de enseñanza y aprendizaje de la asignatura Matemática.

- Los resultados del diagnóstico, en particular, el del dominio de los contenidos matemáticos.

- La necesidad de que los educandos aprendan nuevos conocimientos y habilidades, en particular, los relacionados con la Matemática, y su significatividad.

- La utilización de los diferentes recursos del lenguaje para el logro de una comunicación efectiva, incluyendo el empleo riguroso del lenguaje matemático.

En función de estos problemas profesionales se identifican entonces los siguientes objetivos generales:

- Demostrar dominio del contenido de enseñanza y aprendizaje de la Matemática y de las disciplinas de la carrera para una labor educativa eficaz, flexible e innovadora.

- Dirigir el proceso pedagógico y en particular, el de enseñanza y aprendizaje de la asignatura Matemática, en función de la educación integral de los educandos.

- Fundamentar desde las ciencias de la educación y los contenidos de las disciplinas propias de la carrera, alternativas de solución a los problemas profesionales.

- Identificar y solucionar problemas que surjan en la dirección del proceso pedagógico y, en particular, el de enseñanza y aprendizaje de la Matemática, con la aplicación del método científico y los elementos de la investigación educativa.

- Enseñar a formular y resolver problemas relacionados con diferentes aspectos de la realidad, utilizando contenidos de la matemática, sobre la base de la aplicación de procesos de pensamiento, procedimientos y estrategias de trabajo y el aprovechamiento de las tecnologías de la información y las comunicaciones.

\section{La estrategia didáctica como resultado científico. Fundamentos teóricos}

Al estudiar en la bibliografía especializada las posiciones teóricas que fundamentan el diseño de una estrategia en el contexto educacional y, en particular, de una estrategia didáctica se asumen como esenciales las posiciones de Rodríguez y Rodríguez (2011) al caracterizarla como uno de los tipos de resultados científicos.

Los autores del informe asumen la estrategia:

Como cierto ordenamiento de las acciones en el curso de la resolución de un problema en el que cada paso es necesario para el siguiente. Estas secuencias de acciones están fuertemente orientadas hacia el fin a alcanzar. La persistencia en un procedimiento o su cambio se encuentra también relacionado con el éxito logrado en la consecución de un fin (Rodríguez y Rodríguez, 2011, p. 34).

Igualmente consideran la estrategia didáctica como:

La proyección de un sistema de acciones a corto, mediano y largo plazos los cuales permiten la transformación del proceso de enseñanza-aprendizaje en una asignatura, nivel o institución tomando como 
base los componentes de este y que permite el logro de los objetivos propuestos en un tiempo concreto (Rodríguez y Rodríguez, 2011, p. 39).

Desde estas posiciones se comparten también los criterios que relacionan la estructura de una estrategia. Esta se organiza de la siguiente manera: objetivo general y etapas (diagnóstico, planeación, ejecución y evaluación) contentivas a su vez de objetivos, acciones, orientaciones metodológicas y formas de implementación.

A partir de los aspectos anteriores, los autores del artículo consideran necesario precisar que una estrategia didáctica para enseñar a dirigir el proceso de enseñanza y aprendizaje de la resolución de problemas al estudiantado de la Licenciatura en Educación Matemática debe caracterizarse por la integración de contenidos, el enfoque profesional, el enfoque problémico y el carácter sistémico.

En correspondencia con las posiciones teóricas que se asumen, es pertinente que la estrategia didáctica esté guiada por las siguientes exigencias didácticas:

- Promover el interés por la profesión pedagógica en general y, en particular, por la resolución de problemas y su enseñanza.

- Favorecer la aplicación integrada de los conocimientos, habilidades, capacidades y actitudes profesionales desde todos los componentes del proceso de formación.

- Propiciar el desarrollo de la independencia cognoscitiva a partir de un sistema de tareas docentes variadas y con un gradual nivel de complejidad.

- Estimular el autoaprendizaje del alumnado y la utilización de variadas estrategias de aprendizaje.

- Favorecer el intercambio de experiencias basadas en las relaciones profesionales e interpersonales, en la cooperación y el aprendizaje grupal.

\section{La estrategia didáctica. Resultado del estudio empírico y teórico que se desarrolló}

La estrategia didáctica se diseña para ser utilizada desde el colectivo de la disciplina principal integradora Formación Laboral Investigativa (FLI) en la carrera Licenciatura en Educación Matemática, con la intención de orientar las acciones generales y específicas que le corresponde ejecutar a docentes y estudiantes durante el proceso de formación didáctica; esta tiene en cuenta las implicaciones derivadas de los resultados del diagnóstico descrito en el apartado primero. Además, para su diseño se tuvo en cuenta las exigencias del programa de formación; en particular, el Modelo del Profesional y los programas de las disciplinas del currículo.

Sus acciones, deben considerar los momentos de desarrollo de la disciplina FLI. El primero, antecede a las asignaturas de Didáctica de la Matemática y Metodología de la Investigación Educativa; el segundo, coincide con el desarrollo de las mencionadas asignaturas y el tercero, corresponde a la etapa en que ya cursaron estas materias.

Según Zúñiga (2017), la estrategia didáctica constituye un procedimiento organizado, formalizado y orientado hacia la obtención de una meta. Luego, el objetivo general de esta es perfeccionar el proceso de formación didáctica para la resolución de problemas en estudiantes de la Licenciatura en Educación Matemática.

Para su diseño se plantean objetivos específicos y acciones según las etapas que consideraron Pérez, Valdés y Garriga (2019). Además, se ofrecen orientaciones metodológicas para su implementación según el momento de desarrollo en que se encuentre la disciplina principal integradora FLI. 


\section{Diagnóstico}

Objetivo: Diagnosticar el dominio de los contenidos que se necesitan durante la formación didáctica para la resolución de problemas del estudiantado de la Licenciatura en Educación Matemática.

Acciones:

- Determinación de las dimensiones e indicadores por evaluar en el diagnóstico.

- Determinación de los contenidos por diagnosticar.

- Selección de los métodos y diseño de los instrumentos por utilizar para el desarrollo del diagnóstico de los contenidos.

- Aplicación de los instrumentos por utilizar para la elaboración del diagnóstico del dominio de los diferentes contenidos.

- Procesamiento de la información y valoración de los resultados en el colectivo de la disciplina FLI; así como en los colectivos de disciplina y de año para la proyección de acciones correctivas en función de erradicar las debilidades identificadas.

\section{Orientaciones metodológicas para su instrumentación}

En la tercera tarea del estudio (Pérez et al., 2018) se precisaron las dimensiones e indicadores por utilizar durante el diagnóstico. En correspondencia con la asignatura y el momento de desarrollo de la disciplina FLI, cada docente deberá seleccionar y adaptar los que considere pertinente incluir en los instrumentos de diagnóstico.

Para la determinación de los contenidos a diagnosticar se sugiere tener presente los objetivos de la disciplina y asignatura, considerar el momento de desarrollo de la disciplina principal integradora y centrar la atención en los conocimientos y habilidades matemáticas y didácticas que el estudiantado poseer.

Se recomienda, además, diagnosticar de forma transversal en todas las asignaturas los siguientes elementos: el desarrollo de la habilidad resolver problemas y el empleo de los procedimientos heurísticos.

Los métodos por utilizar deben ser variados; se recomiendan, la observación del desempeño, la revisión del producto de la actividad y la entrevista a estudiantes. El diseño de los instrumentos y su aplicación debe tener como centro la búsqueda de la información relacionada con el nivel que logra el alumnado para dirigir el proceso de enseñanza y aprendizaje de la resolución de problemas. En las asignaturas que tributan a la formación matemática, se debe profundizar en el desarrollo de la habilidad resolver problemas y en las estrategias que utiliza el estudiantado con este fin.

Los resultados del diagnóstico se deben analizar y valorar en los diferentes niveles de trabajo metodológico, siempre precisando las fortalezas y debilidades en que debe sustentarse la formación didáctica del conjunto estudiantil para la resolución de problemas. Sobre este particular, se precisa la importancia de hacer partícipe al alumnado para que comprenda sus dificultades, seleccione las estrategias de aprendizaje por utilizar y las acciones por ejecutar para erradicarlas con el profesorado.

\section{Planeación}

Objetivo: Diseñar las acciones que demanda la formación didáctica del estudiantado para la resolución de problemas.

Las acciones de esta etapa forman parte de la planificación a largo, mediano y corto plazo y sus resultados, a juicio de los investigadores, deben quedar plasmados en el programa y en el plan calendario de la asignatura; así como en cada una de las formas de organización que se decida utilizar. 


\section{Acciones por realizar:}

- Determinación de los problemas profesionales y de los objetivos generales a cumplir desde cada asignatura a partir del estudio de los documentos rectores.

- Determinación de los contenidos que fundamentan la formación didáctica para la resolución de problemas o aquellos con potencialidades de contribuir a esta.

- Selección de los métodos y los medios que más favorecen la formación didáctica para la resolución de problemas.

- Determinación de los tipos de tareas docentes a utilizar desde los tres componentes del proceso de formación profesional.

- Diseño de las tareas docentes a proponer para los tres componentes del proceso de formación profesional y las formas de organización a utilizar.

- Determinación de las vías de evaluación y diseño de los instrumentos que se utilizarán para evaluar la formación didáctica para la resolución de problemas.

\section{Orientaciones metodológicas para su instrumentación}

Para la determinación de los problemas profesionales y de los objetivos generales de la carrera a cumplir en cada asignatura se sugiere estudiar los siguientes documentos: el Modelo del Profesional, el programa de la disciplina FLI el programa de la disciplina al que pertenece la asignatura y los objetivos del año.

$\mathrm{Al}$ analizar el Modelo del Profesional debe centrarse la atención, primero en los problemas profesionales y, segundo, en los objetivos generales. En ambos casos, es preciso identificar la relación de estos con la formación didáctica para la resolución de problemas y lograr su derivación al año académico y a la asignatura. De forma similar, se debe proceder al estudiar el programa de la disciplina al que pertenece la asignatura y sus relaciones deberán quedar expresadas en el programa de la asignatura.

También es recomendable que al estudiar el programa de la disciplina FLI se determinen los tipos de tareas que más se ajustan a los propósitos del año, de la asignatura y de la formación didáctica para la resolución de problemas. En él se sugieren 13 tipos de tareas por desarrollar por el estudiantado de las cuales siete se relacionan directamente con el objetivo de esta investigación.

En cada asignatura, desde la propia elaboración del programa se deben determinar los contenidos que fundamentan o aportan a la formación didáctica para la resolución de problemas; es decir: conocimientos, habilidades, hábitos y actitudes matemáticas o didácticas que serán introducidas o sistematizadas según el año académico y el nivel de desarrollo del alumnado. Las asignaturas de formación matemática deben resaltar los contenidos que contribuyen al logro del objetivo general de la estrategia didáctica.

Se sugiere que en todas las asignaturas se asuma la clasificación de los métodos según el carácter de la actividad cognoscitiva (Labarrere y Valdivia, 1988): explicativo-ilustrativo, reproductivo, exposición problémica, búsqueda parcial, conversación heurística e investigativo. De esta forma, se estarían ofreciendo modos de actuación y desarrollando en el estudiantado las formas de trabajo y de pensamiento más afines a la resolución de problemas. También, se recomienda utilizar según las potencialidades de los contenidos, las diferentes tendencias para la resolución de problemas, de esta forma se utilizarían problemas con diferentes intenciones didácticas.

Los medios de enseñanza y aprendizaje deberán corresponderse con los métodos que se seleccionen; además es pertinente insistir en el cumplimiento de uno de los lineamientos de la asignatura matemática: la utilización de los asistentes con fines heurísticos, o como herramienta para el cálculo y la representación.

Los tipos de tareas docentes que cada asignatura determine deben abarcar los tres componentes del proceso de formación profesional y estarán encaminadas en dos direcciones fundamentales: las relacionadas con la formación matemática (específicamente orientadas hacia la resolución de problemas) y las que se relacionan 
con la formación didáctica propiamente dicha (aunque estas incluyen la anterior). En general, todas las asignaturas deberán considerar los tipos de tareas identificadas desde el estudio del programa de la disciplina principal integradora.

El diseño de las tareas debe expresar con claridad el objetivo y su aporte a la formación didáctica para la resolución de problemas, además de las acciones por efectuar, los medios que deben ser utilizados y las formas de control. Se sugiere, además, que las tareas que se diseñen logren la integración de los contenidos que aporten al objetivo de la investigación; de esta forma se propiciará entonces su sistematización y contextualización, así como el intercambio del estudiantado durante su ejecución.

Las tareas para el componente académico estarán dirigidas fundamentalmente a: resolver y argumentar la vía de solución de los problemas de las diferentes disciplinas con la utilización de los recursos heurísticos disponibles, formular problemas, explicar el tratamiento metodológico de los problemas escolares, en dependencia del momento de desarrollo de la disciplina FLI en el cual se ubique la asignatura y a planificar el proceso de enseñanza y aprendizaje de la resolución de problemas.

Las tareas del componente laboral deberán enfatizar básicamente en la resolución de los problemas de los textos escolares y en las acciones propias de la formación didáctica para la resolución de problemas.

En las tareas del componente investigativo se debe priorizar la valoración de las acciones del proceso de enseñanza y aprendizaje de la resolución de problemas y en el diseño de alternativas de solución a las dificultades que se identifiquen; tanto, en los procederes didácticos del cuerpo docente, como en el nivel de desarrollo de la habilidad resolver problemas y en las estrategias de aprendizaje que utiliza el alumnado.

Los tipos fundamentales del trabajo investigativo que desarrollan estudiantes universitarios son el trabajo de curso, el trabajo de diploma y el trabajo investigativo extracurricular (MES, 2018). Para los fines de esta investigación el énfasis debe ponerse en aprovechar eficientemente las posibilidades que ofrecen los trabajos extracurriculares y los de cursos vinculados a las asignaturas.

Para la determinación de las vías de evaluación es preciso tener en cuenta que la evaluación del aprendizaje en la educación superior tiene carácter continuo, cualitativo e integrador y se basa, esencialmente, en el desempeño del alumnado durante el proceso de enseñanza y aprendizaje (Horruitiner, 2007), los tipos y formas de evaluación reconocidas para este nivel de enseñanza y que estas deben articular coherentemente lo teórico y lo práctico; así como lo matemático y lo didáctico. El diseño de los instrumentos debe propiciar la integración de los contenidos correspondientes a la formación didáctica para la resolución de problemas.

\section{EJECUCIÓN}

Objetivo: Ejecutar las acciones diseñadas con el propósito de lograr la formación didáctica del estudiantado para la resolución de problemas.

Dado el carácter de sistema de las etapas, en la explicación de las acciones de las etapas anteriores se ofrecen sugerencias para su ejecución. A continuación, se precisan y explican las que desarrollarían docentes y estudiantes durante el proceso de enseñanza y aprendizaje de cada asignatura en particular.

\section{Acciones para el cuerpo docente:}

- Elaboración de medios y materiales didácticos para favorecer la formación didáctica para la resolución de problemas.

- Orientación de las tareas docentes por ejecutar en los tres componentes del proceso de formación profesional.

- Control del aprendizaje del estudiantado durante la elaboración de las tareas docentes.

Acciones para el estudiantado:

- Identificación de las estrategias de aprendizaje y de las técnicas para la resolución de problemas matemáticos. 
- Resolución y formulación de problemas de la matemática escolar y superior.

- Explicación de las vías de solución utilizadas para resolver los problemas de la matemática escolar y superior.

- Valoración de las vías utilizadas por estudiantes de los diferentes niveles educativos para resolver los problemas matemáticos.

- Identificación de los procedimientos heurísticos (principios, reglas y estrategias) a utilizar durante la resolución de problemas.

- Identificación de las fases del programa heurístico general y las acciones correspondientes a cada una de ellas.

- Desarrollo del tratamiento metodológico de problemas a partir de la aplicación del programa heurístico general.

- Diseño de sistemas de clase donde se enfatice en la utilización de los problemas con diferentes intenciones didácticas.

- Planificación e impartición de clases donde se de tratamiento a los problemas con diferentes intenciones didácticas.

\section{Orientaciones metodológicas para la instrumentación de las acciones del profesorado}

Se recomienda que, a partir del análisis didáctico de los contenidos de cada asignatura y de la identificación de sus relaciones con la formación didáctica para resolución de problemas, las y los docentes elaboren los medios y materiales didácticos que pueden favorecer la formación didáctica para la resolución de problemas.

Entre los más importantes se consideran las guías de estudio, láminas (en papel o digitales) con resúmenes o esquemas que relacionen las estrategias y técnicas para la resolución de problemas, láminas (en papel o digitales) con resúmenes o esquemas que relacionen los procedimientos heurísticos y los artículos que contribuyan a la actualización de la bibliografía de las asignaturas.

$\mathrm{Al}$ orientar las tareas docentes se debe precisar con suficiente claridad el objetivo y explicar su aporte a la formación didáctica para la resolución de problemas, las acciones que deberán ejecutarse para su cumplimiento, la bibliografía, los medios por utilizar; así como la forma de control.

Durante el desarrollo de las tareas, el personal docente deberá observar el desempeño estudiantil, hacer las aclaraciones y ofrecer los impulsos pertinentes. De igual manera, deberán estimular el intercambio de experiencias, el trabajo cooperado y el empleo sistemático e intencional de las formas de trabajo y de pensamiento matemático; así como de los procedimientos heurísticos.

En el proceso de ejecución de las tareas del componente laboral o investigativo, el grupo docente debe insistir en los modos de actuación profesional a lograr, en la sistematización y la contextualización de los contenidos matemáticos y didácticos, a la vez que propician el intercambio con profesores de experiencia y con estudiantes de los diferentes niveles educativos.

Por otra parte, mientras el profesorado da seguimiento a las actividades estudiantiles durante la elaboración de las tareas, se sugiere que recopile información cualitativa que le permita obtener juicios valorativos sobre su aprendizaje. Puede utilizar para ello la observación del desempeño, la revisión del producto de la actividad y el propio intercambio oral con estudiantes y entre estos últimos.

\section{Orientaciones metodológicas para la instrumentación de las acciones del estudiantado}

El alumnado deberá, desde las asignaturas de formación matemática, desarrollar actividades donde identifiquen las estrategias de aprendizaje y las técnicas para la resolución de problemas. En este sentido, se les sugiere que elaboren un resumen o un material didáctico donde puedan disponer de la mayor cantidad 
posible; este podrá ser enriquecido sistemáticamente y completar, posteriormente, desde la Didáctica de la Matemática y, a partir, de las sesiones de práctica laboral.

Las estrategias de aprendizaje y las técnicas para la resolución de problemas, las pueden obtener o sistematizar a partir de la propia resolución de problemas, del intercambio con docentes en las asignaturas o con estudiantes y docentes de experiencia de los diferentes niveles de educación.

Otra acción que deberán ejecutar de forma sistemática es la resolución y formulación problemas de la matemática escolar y superior, para así desarrollar la habilidad propiamente dicha y sus modos de actuación profesional. En este sentido, se insiste en que siempre expliquen la vía de solución utilizada por ellos.

Se recomienda que siempre que los contenidos de las asignaturas lo permitan el estudiantado resuelva todos los problemas que se proponen en los textos escolares y que organicen sesiones de análisis y valoración donde destaquen las vías de solución utilizadas y entre ellas, las más ventajosas.

Igualmente procederán con los problemas de la matemática superior que se le oriente resolver en las diferentes asignaturas. Del primero de los casos, puede derivarse un material de apoyo a la docencia que a futuro les resulte útil durante su desempeño profesional.

Al trabajar la formulación de problemas, tanto en las asignaturas de formación matemática como en la Didáctica de la Matemática y en las sesiones de práctica laboral, se deben atender tres aspectos esenciales: el empleo adecuado de los objetos y datos matemáticos, el uso adecuado de la lengua materna y que se cumplan todas las acciones que didácticamente incluye la actividad de formulación en sí misma. En este caso, se recomienda que se precise en cada caso la intención didáctica con que puedan ser utilizados los problemas formulados.

Se sugiere que en las actividades del componente laboral e investigativo donde se resuelvan problemas se preparen debates o intercambios donde sean valoradas las vías de solución que utiliza el estudiantado.

Otra actividad que deben desarrollar sistemáticamente es la identificación de los procedimientos heurísticos (principios, reglas y estrategias) que se utilizan al resolver los problemas; primero, desde las asignaturas de formación matemática a partir de la actuación de las y los docentes y segundo, desde la Didáctica de la Matemática y las sesiones de práctica laboral. En todos los casos, se le recomienda que elaboren un resumen que deberá ser enriquecido con sus propias experiencias de aprendizaje.

También, deberán entrenarse en la identificación de las fases del programa heurístico general y las acciones por ejecutar en cada una de ellas; es decir, los principios, reglas y estrategias que se utilizarán para llegar a la vía de solución más correcta. Esta actividad la deben efectuar desde todas las asignaturas donde se resuelven problemas.

El desarrollo del análisis metodológico de problemas de la matemática escolar a partir del empleo de los procedimientos heurísticos (principios, reglas y estrategias) y de la aplicación del programa heurístico general es una actividad ya propiamente de la Didáctica de la Matemática y que se puede organizar también en las sesiones de práctica laboral. En este sentido, se recomienda que el alumnado resuelva los problemas y, a su vez, destaque los posibles recursos de la heurística que emplea o que emplearía para el trabajo en el aula. Para esta actividad es pertinente considerar la intención didáctica con que serán utilizados los problemas en clases, de eso dependerá el rol del profesorado y del estudiantado.

Para el diseño de los sistemas de clases, actividad que podrán desarrollar para cualesquiera de los contenidos escolares, se precisa que el grupo de estudiantes determine en cada clase los problemas por utilizar y su intención didáctica. En las clases propiamente de resolución de problemas (donde la intención didáctica es la fijación), deberán explicitarlo desde la formulación del objetivo, el método y los posibles procedimientos a emplear; así como en las tareas que serán propuestas para cada momento. En las clases donde se utilicen problemas con una intención didáctica diferente, se sugiere precisar cuál y como los utilizarán.

Durante las tareas asociada a la planificación de clases donde se utilicen problemas se sugiere que estos sean resueltos y se acompañen de un análisis metodológico que explicite la utilización de los procedimientos 
heurísticos y de las formas de trabajo y de pensamiento matemático; aspecto este que permitirá luego enseñar coherentemente a estudiantes para hacerlo en clase.

\section{Evaluación}

Objetivo: Evaluar la calidad con que se realizan las acciones de la estrategia y su efectividad en el logro de la formación didáctica de las y los estudiantes para la resolución de problemas.

En este caso, se explicita el carácter de sistema de las etapas de la estrategia didáctica y además el propio carácter de proceso que caracteriza la evaluación. De ahí, que en cada momento se efectúen acciones que aportan juicios valorativos relacionados con la calidad de las acciones de la estrategia y con su efectividad en la práctica. No obstante, en esta etapa se precisan y explican las más generales.

\section{Acciones:}

- Autoevaluación del proceso seguido durante la elaboración del diagnóstico, la planeación y la ejecución de las acciones.

- Realización de sesiones en profundidad entre los miembros de los diferentes colectivos metodológicos para el intercambio y la socialización de las mejores experiencias derivadas del proceso de formación didáctica.

- Observación del desempeño de las y los estudiantes durante la ejecución del proceso de enseñanzaaprendizaje de la resolución de problemas.

- Revisión del producto de la actividad que se deriva de la planificación del proceso de enseñanza y aprendizaje de la resolución de problemas.

- Elaboración de pruebas pedagógicas (teóricas y prácticas) centradas en el desarrollo de la habilidad resolver problemas y en la formación didáctica para la resolución problemas.

- Valoración de los resultados de los métodos e instrumentos que se apliquen.

- Diseño de acciones correctivas o actualización de las acciones según los resultados que se obtienen en cada momento.

\section{Orientaciones metodológicas para su implementación}

Las dos primeras acciones se enfocan hacia la evaluación de la calidad de las acciones de la estrategia; aunque pudieran ser usados otros métodos, los propuestos pueden ofrecer información certera sobre el resultado de cada una de ellas y criterios que fundamenten el rediseño, la actualización o la inclusión de nuevas acciones.

Las acciones de la tres a la cinco centran su atención en la evaluación del nivel de formación didáctica del estudiantado para la resolución de problemas. En cada caso el diseño deberá tener en cuenta los indicadores a medir según el año en que se encuentre el alumnado. Se recomienda que sean instrumentos integradores diseñados desde el colectivo de año, con la participación de docentes de todas las asignaturas que han tributado al proceso de formación didáctica.

Es importante que en todas las acciones anteriores se logre una participación de docentes y estudiantes; así como de otros agentes que intervienen en el proceso de formación didáctica para la resolución de problemas.

Para la valoración de los resultados se recomienda centrar la atención en la información que aporte elementos relacionados con la calidad de las acciones y sobre el nivel de formación didáctica logrado por el alumnado. Esta acción debe concluir en cada momento con la identificación o actualización de las fortalezas y debilidades que caracterizan el proceso de formación didáctica para la resolución de problemas.

A partir de las fortalezas y debilidades que sean identificadas se recomienda que se diseñen acciones correctivas en función de reajustar lo desarrollado como parte del proceso de formación didáctica y del nivel 
logrado en el estudiantado. Por otra parte, es pertinente la actualización de acciones o la inclusión de otras que favorezcan los resultados obtenidos.

\section{Conclusiones}

Resultan importantes fundamentos teóricos para el diseño de la estrategia didáctica integradora de las posiciones que se asumen en relación con la categoría formación didáctica, al proceso de formación didáctica y sus exigencias en la carrera Licenciatura en Educación Matemática. Igualmente, se consideraron los problemas profesionales, objetivos generales y las tareas precisadas en el modelo del profesional de la carrera antes mencionada.

Las fortalezas y debilidades identificadas como resultado del diagnóstico del estado actual del proceso de formación didáctica del estudiantado de la Licenciatura en Educación Matemática para la resolución de problemas constituyeron un importante referente para el diseño de la estrategia didáctica integradora.

La estrategia didáctica integradora elaborada ofrece un sistema de acciones que permite perfeccionar el proceso de formación didáctica de estudiantes de la Licenciatura en Educación Matemática para la resolución de problemas. Esta ha considerado las etapas de diagnóstico, planeación, ejecución y evaluación; para cada una de ellas, se precisa el objetivo, las acciones a ejecutar y las orientaciones metodológicas para su implementación.

\section{ReFERENCIAS BibLiográficAS}

Almeida, B. A. (2000). La integración de los componentes organizacionales del proceso docente educativo en la evaluación del aprendizaje(Tesis de maestría). Instituto Superior Pedagógico "Enrique José Varona”, La Habana: Cuba.

Ball, D. L.; Thames, M. H.; Phelps, G. (2008). Content knowledge for teaching: What makes it special? Journal of Teacher Education, 59(5), 89-407.

Ballester, S., Santana, H., Hernández, S., Cruz, I., Arango, C., García, M., ... Torres, P. (1992). Metodología de la enseñanza de la Matemática. (tomo I). La Habana: Cuba: Editorial Pueblo y Educación.

Beltrán, C. (2012). La estructuración sistémica del contenido como base para la gestión de competencias profesionales pedagógicas desde la disciplina Didáctica de la Matemática (Tesis de doctorado). Universidad de Ciencias Pedagógicas “José de la Luz y Caballero”, Guantánamo, Cuba.

Cooney, T. J. (1986). The Contribution of Theory to Mathematics Teacher Education. Paper Prepared for Sesions on Theory in Mathematics Education. Adelaida: V ICME.

Feria, F. F. (2003). El perfeccionamiento de la dinámica del proceso docente educativo en la disciplina Metodología de la Enseñanza de la Matemática (Tesis de doctorado). Instituto Superior Pedagógico "José de la Luz y Caballero", Holguín: Cuba.

Fernández, J. (1999). Una propuesta para perfeccionar el componente académico en la disciplina Metodología de la Enseñanza de la Matemática (Tesis de maestría). Instituto Superior Pedagógico "Enrique José Varona”, La Habana: Cuba.

Flores, P. y Penas, M. (2003). Formación inicial de profesores de matemáticas reflexivos". Revista Educación y Pedagogía, 25(35), $95-116$.

Garcés, W. (2003). El sistema de tareas como modelo de actuación didáctica en la formación de profesores de MatemáticaComputación (Tesis de doctorado). Instituto Superior Pedagógico "José de la Luz y Caballero", Holguín: Cuba.

Godino, J. D. (2002). La formación matemática y didáctica de maestros como campo de acción e investigación para la didáctica de las matemáticas. Granada: Proyecto Edumat-Maestros. Universidad de Granada. Recuperado de h ttps://bit.ly/3aU6nxC 
Godino, J. (2009). Categorías de análisis de los conocimientos del profesor de Matemáticas. Revista Iberoamericana de Educación Matemática, (20), 13-31. Recuperado de http://www.fisem.org/www/union/revistas/2009/20/Un ion_020_007.pdf

Godino, J. D., (2011). Indicadores de idoneidad didáctica de procesos de enseñanza y aprendizaje de las matemáticas. En XIII Conferência Interamericana de Educação Matemática (CIAEM-IACME), Recife: Brasil.

Godino, J. D. y Batanero, C. (2003). Fundamentos de la enseñanza y el aprendizaje de las matemáticas para maestros. Granada: Proyecto Edumat-Maestros. Universidad de Granada. Recuperado de https://bit.ly/39PN5cO

Godino, J. D. y Batanero, C. (2008). Desarrollo de competencias para el análisis didáctico del profesor de matemáticas. En Actas de las VI Jornadas de Educación Matemática Región de Murcia, Murcia. Recuperado de http:xiii.ciaemredumate.org/index.php

Gómez, B. (2000). ¿Qué aporta la Didáctica de la Matemática a la formación inicial de los matemáticos? Tomo 2. Chile: RELME. Recuperado de www.uv.es/gomezb/23Queaportaladidmat.pdf

González, F. E. (2000). Los nuevos roles del profesor de Matemática. Retos de la Formación de Docentes para el siglo XXI. Revista Paradigma, 21, 1-20.

Gort, M. (1999). Una propuesta para perfeccionar el componente laboral en la disciplina Metodología de la Enseñanza de la Matemática (Tesis de maestría). Instituto Superior Pedagógico "Enrique José Varona”, La Habana, Cuba.

Horruitiner, P. (2007). Una nueva generación de planes de estudio. Pedagogía Universitaria, 12(4), 133-157. Recuperado de http://cvi.mes.edu.cu/peduniv/index.php/peduniv

Labarrere, G. y Valdivia, G. (1988). Pedagogía. La Habana: Pueblo y Educación.

Llinares, S. y Krainer, K. (2006). Mathematics (students) teachers and teacher educators as learners. En Gutiérrez, A. y Boero, P. (Eds), Handbook of Research on the Psychology of Mathematics Education: Past, Present and Future (pp. 429-459). Rotterdam: Sense Publishers.

Ministerio de Educación Superior de Cuba [MES]. (2016). Modelo del Profesional. Plan de Estudio E. Carrera Licenciatura en Educación Matemática. Cuba: La Habana. Material en soporte digital.

Ministerio de Educación Superior de Cuba [MES]. (2018). Reglamento del Trabajo Docente y Metodológico en la Educación Superior. Resolución Ministerial 2. Cuba: La Habana. Material en soporte digital.

Oliveras, M. L. (1997). Reflexiones sobre el profesor de Matemáticas del Próximo Siglo y Análisis de Proposiciones Curriculares en esa Dirección. En Olfos, R. y González, H. (Eds.) Alternativas para la Formación de Profesores de Matemática. Seminario Internacional sobre Formación de Profesores de Matemática. (pp. 49-74). La Serena, Chile: Edición conjunta de la Sociedad Chilena de Educación Matemática y el Departamento de Matemática de la Universidad de la Serena.

Otte, M. (1979). Formación y vida profesional de los profesores de Matemática. En UNESCO (Ed.) Revista Nuevas Tendencias de la Enseñanza de la Matemática (pp. 119-150). Montevideo, Uruguay: UNESCO

Pérez, A. (2015). La integración de las invariantes de la habilidad profesional planificar el proceso de enseñanzaaprendizaje desde la Didáctica de la Matemática (Tesis de doctorado). Universidad “José Martí Pérez". Cuba.

Pérez, A., Garriga, A. T, Pérez, A. A., Rodríguez, N. C., Rodríguez, L., Fonseca, A. L,..., Bravo, J. L. (2018). Diagnóstico del nivel de formación didáctica que logran los estudiantes de la Licenciatura en Educación Matemática-Física y Licenciatura en Matemática para enseñar a resolver problemas. Universidad “José Martí Pérez”. Cuba.

Pérez, A., Pérez, A. A., Rodríguez, N. C., Rodríguez, L., Fonseca, A. L, Quero, O.,... Bravo, J. L. (2017a). La formación didáctica para enseñar a resolver problemas matemáticos. Instrumentos para su evaluación. En I. Ramos, D. Echemendía y Y. Pérez (Comp.), Dimensiones, indicadores e instrumentos para comprobar el nivel de preparación del futuro profesional universitario para enseñar a resolver problemas (pp. 110-124). Cuba: Universidad de Sancti Spíritus “José Martí Pérez”.

Pérez, A., Rodríguez, N. y Quero, O. (2017b). La formación didáctica del profesor de Matemática para la resolución de problemas. Exigencias actuales. En CD-ROOM, Memorias del VI Intercambio entre Educadores De Latinoamérica. Pinar del Río.

Pérez, A., Valdés, M., y Garriga, A. (2019). Estrategia didáctica para enseñar a planificar el proceso de enseñanzaaprendizaje de la Matemática. Revista Educación. 43(2) 1-18. 
Pino-Fan, L. y Godino, J. (2015). Perspectiva ampliada del conocimiento didáctico-matemático del profesor. Paradigma, 36(1), 87-109.

Rico, L. (1992). Proyecto docente. Granada, España: Universidad de Granada.

Rodríguez, M. A. y Rodríguez, A. (2011). La estrategia como resultado científico de la investigación educativa. En N. De Armas y A. Valle. (Ed.). Resultados cientificos en la investigación educativa. (pp. 22-40). La Habana, Cuba: Pueblo y Educación.

Schoenfeld, A. y Kilpatrick, J. (2008). Towards a theory of profiency in teaching mathematics. En D. Tirosh, y T. L. Wood (Eds.), Toolsand processes in mathematics teacher education. (pp. 321-354). Rotterdam: Sense Publishers.

Shulman, L. (1986). Those who understand: Knowledge growth in teaching. Educational Researcher, 15(2), 4-14.

Sierra, T., Bosch, M. y J. Gascón. (2012). La formación matemático-didáctica del maestro de Educación Infantil: el caso de «cómo enseñar a contar. Revista de Educación, (357), 51-256.

Zuñiga, M. (2017). La estrategia didáctica: Una combinación de técnicas didácticas para desarrollar un plan de gestión de riesgos en la clase. Revista Educación, 41(1), 1-18.

\section{BY-NC-ND}

